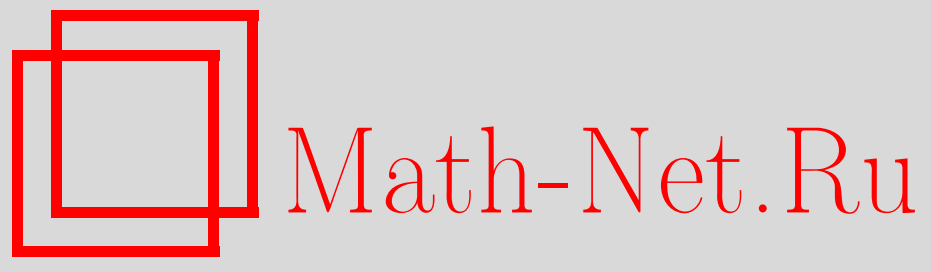

В. М. Бухштабер, $f$-полиномы простых многогранников и двупараметрический род Тодда, УМH, 2008, том 63, выпуск 3, 153-154

DOI: https://doi.org/10.4213/rm9211

Использование Общероссийского математического портала Math-Net.Ru подразумевает, что вы прочитали и согласны с пользовательским соглашением http://www . mathnet.ru/rus/agreement

Параметры загрузки:

IP : 54.80 .97 .219

26 апреля 2023 г., 15:15:22

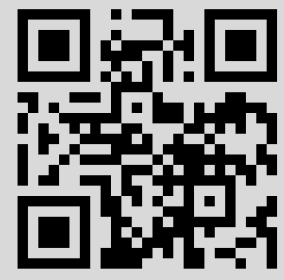




\section{$f$-полиномы простых многогранников и двупараметрический род Тодда}

\section{В. М. Бухштабер}


вершине сходится в точности $n$ гиперграней. Сведения о простых многогранниках см. в [1], [2]. Два многогранника называются комбинаторно эквивалентными, если существует взаимно однозначное соответствие между их множествами граней, сохраняющее отношение включения. Класс комбинаторно эквивалентных выпуклых многогранников называется комбинаторным многогранником.

Обозначим через $\mathscr{P}_{2 n}$ абелеву группу, порожденную комбинаторными простыми $n$-мерными многогранниками, в которой сумма соответствует несвязному объединению многогранников, а нуль - пустому множеству.

Прямое произведение $P_{1}^{n} \times P_{2}^{m}$ простых многогранников $P_{1}^{n}$ и $P_{2}^{m}$ также является простым многогранником.

Через $\mathscr{P}=\sum_{2 n \geqslant 0} \mathscr{P}_{2 n}$ обозначим градуированное коммутативное ассоциативное кольцо, в котором умножение соответствует прямому произведению простых многогранников, а единицей является $P^{0}=$ (точка). Каждая грань простого многогранника является простым многогранником и пересечением в точности $(n-k)$ гиперграней.

Лемма 1. Линейный оператор $d: \mathscr{P} \rightarrow \mathscr{P}$, сопоставляющий простому многограннику $P^{n}$ несвязное объединение всех его гиперграней, является дифференцированием кольиа $\mathscr{P}$.

ОПРЕДЕлЕниЕ 2. f-полиномом (face-polynomial) простого многогранника $P^{n}$ называется однородный полином

$$
f\left(P^{n}\right)=\alpha^{n}+f_{n-1,1} \alpha^{n-1} t+\cdots+f_{1, n-1} \alpha t^{n-1}+f_{0, n} t^{n},
$$

где $f_{k, n-k}$ - число граней размерности $k$ в $P^{n}$.

Теорема 3. 1) Линейное отображение $f: \mathscr{P} \rightarrow \mathbb{Z}[\alpha, t]$, заданное формулой (1), является кольчевым гомоморфизмом таким, что

$$
f\left(d P^{n}\right)=\frac{\partial}{\partial t} f\left(P^{n}\right) .
$$

2) Пусть $F: \mathscr{P} \rightarrow \mathbb{Z}[\alpha, t]$ - линейное отображение такое, что $\left.F\left(P^{n}\right)\right|_{t=0}=\alpha^{n}$ u $F\left(d P^{n}\right)=\frac{\partial}{\partial t} F\left(P^{n}\right)$. Тогда $F\left(P^{n}\right)=f\left(P^{n}\right)$.

Теорема 4. $f\left(P^{n}\right)(\alpha, t)=f\left(P^{n}\right)(-\alpha, \alpha+t)$, где $P^{n}-$ простой многогранник.

Соотношение (2) позволяет провести доказательство этой теоремы индукцией по $n$ и получить вывод соотношений Дена-Соммервиля из формулы для эйлеровой характеристики комбинаторной сферы $S^{n-1}=\partial P^{n}$.

Положим $h\left(P^{n}\right)(\alpha, t)=f\left(P^{n}\right)(\alpha-t, t)$.

СледСтвие 5. 1) $h\left(P^{n}\right)(\alpha, t)=h\left(P^{n}\right)(t, \alpha)$.

2) Образ кольчевого гомоморфизма $h: \mathscr{P} \rightarrow \mathbb{Z}[\alpha, t]$, заданного формулой $P^{n} \rightarrow$ $h\left(P^{n}\right)(\alpha, t)$, порожден полиномами $h\left(\Delta^{1}\right)=\alpha+t u h\left(\Delta^{2}\right)=\alpha^{2}+\alpha t+t^{2}$.

3) Пусть $H: \mathscr{P} \rightarrow \mathbb{Z}[\alpha, t]$ - такое линейное отображение, что $\left.H\left(P^{n}\right)\right|_{t=0}=\alpha^{n}$ u $H\left(d P^{n}\right)=\left(\frac{\partial}{\partial \alpha}+\frac{\partial}{\partial t}\right) H\left(P^{n}\right)$. Тогда $H\left(P^{n}\right)=h\left(P^{n}\right)$.

Работа выполнена при поддержке РФФИ (гранты № 08-01-00541, 08-01-91855-КО) и программы "Ведущие научные школы" (проект НШ-1824.2008.1). 
О сериях простых многогранников см. в [3]. Рассмотрим серию многогранников Сташефа (ассоциэдров) $\mathscr{A} \mathrm{s}=\left\{\mathrm{As}^{n}=K_{n+2}, n \geqslant 0\right\}$ и положим $U(\alpha, t, x ; \mathscr{A} \mathrm{s})=$ $\sum_{n \geqslant 0} f\left(\mathrm{As}^{n}\right) x^{n+2}$. Каждая гипергрань многогранника $\mathrm{As}^{n}$ имеет вид $\mathrm{As}^{i} \times \mathrm{As}^{j}$, где $i \geqslant 0, i+j=n-1$. Следовательно, $d \mathrm{As}^{n}=\sum_{i+j=n-1}(i+2)\left(\mathrm{As}^{i} \times \mathrm{As}^{j}\right)$ и $\frac{\partial}{\partial t} f\left(\mathrm{As}^{n}\right)=$ $\sum_{i+j=n-1}(i+2) f\left(\mathrm{As}^{i}\right) f\left(\mathrm{As}^{j}\right)$.

Теорема 6 [4]. Ряд $U(t, x)=U(\alpha, t, x ; A$ s) является решением уравнения Хопба

$$
\frac{\partial}{\partial t} U(t, x)=U(t, x) \frac{\partial}{\partial x} U(t, x)
$$

с начальным условием $U(0, x)=x^{2} /(1-\alpha x)$, следовательно, удовлетворяет квадратному уравнению

$$
t(\alpha+t) U^{2}-(1-(\alpha+2 t) x) U+x^{2}=0 .
$$

Рассмотрим серию многогранников Ботта-Таубса (циклоэдров) $\mathscr{C} \mathrm{y}=\left\{\mathrm{C}^{n}, n \geqslant 0\right\}$ и положим $U(\alpha, t, x ; \mathscr{C} \mathrm{y})=\sum_{n \geqslant 0} f\left(C \mathrm{y}^{n}\right) x^{n}$. Имеем: $d \mathrm{C}^{n}=(n+1) \sum_{i+j=n-1}\left(\mathrm{C}^{i} \times \mathrm{As}^{j}\right)$.

ТеОрема 7. Функция $U(\alpha, t, x ; \mathscr{C}$ у) является решением уравнения

$$
\frac{\partial}{\partial t} U(\alpha, t, x ; \mathscr{C} \mathrm{y})=\frac{\partial}{\partial x}(U(\alpha, t, x ; \mathscr{C} \mathrm{y}) U(\alpha, t, x ; \mathscr{A} \mathrm{s}))
$$

с начальным условием $U(\alpha, 0, x ; \mathscr{C}$ у $)=1 /(1-\alpha x)$.

ЗАмечание 8 . Пусть $U=U_{0}+\mu U_{1}+\left(\mu^{2}\right)$ - решение уравнения Бюргерса $U_{t}=$ $U U_{x}-\mu U_{x x}$. Тогда $U_{0}$ - решение уравнения (3), а $U_{1}-$ решение уравнения $\left(U_{1}\right)_{t}=$ $\left(U_{0} U_{1}\right)_{x}-\left(U_{0}\right)_{x x}$, однородной частью которого является уравнение $(4)$.

Пусть $M^{2 n}$ - компактное гладкое симплектическое многообразие с эффективным гамильтоновым действием компактного тора $T^{n}$. Рассмотрим отображение моментов $\Phi: M^{2 n} \rightarrow \mathbb{R}^{n}$. Получаем простой многогранник $P^{n}=\Phi\left(M^{2 n}\right)=\operatorname{Im} \Phi$. Многообразие $M^{2 n}$ является почти комплексным.

Пусть $\mathrm{Td}_{a, b}$ - двупараметрический род Тодда, соответствующий формальной группе $(u+v-a u v) /(1-b u v)$.

Tеорема 9. $f\left(\Phi\left(M^{2 n}\right)\right)=\gamma \operatorname{Td}_{a, b} M^{2 n}$, где $\gamma$ - замена переменных $a \rightarrow \alpha+2 t$, $b \rightarrow \alpha t+t^{2}$.

СледСтвие 10. Пусть $s_{1}$ - операция Ландвебера-Новикова в комплексных кобордизмах. Тогда $\frac{\partial}{\partial t} f\left(\Phi\left(M^{2 n}\right)\right)=\gamma \operatorname{Td}_{a, b}\left(s_{1}\left[M^{2 n}\right]\right)$.

Используя связь $\mathrm{Td}_{a, b}$ с характеристическими числами, получаем известные формулы: для старшего числа Черна $c_{n}\left(M^{2 n}\right)=f_{0, t} t^{n}$; для рода Тодда $\operatorname{Td}\left(M^{2 n}\right)=\alpha^{n}$; для сигнатуры $\sigma\left(M^{2 n}\right)=(-1)^{n}\left[2^{n}-2^{n-1} f_{n-1,1}+\cdots+(-1)^{n-1} \cdot 2 f_{1, n-1}+(-1)^{n} f_{0, n}\right] t^{n}$.

Частично результаты этой работы опубликованы в [5].

\section{Список литературы}

[1] V. M. Buchstaber, T. E. Panov, Torus actions and their applications in topology and combinatorics, Univ. Lecture Ser., 24, Amer. Math. Soc., Providence, RI, 2002. [2] B. M. Бухштабер, Т.Е. Панов, Торические действия в топологии и комбинаторике, МЦНМО, М., 2004. [3] A. Postnikov, V. Reiner, L. Williams, Faces of generalized permutohedra, arXiv: math/0609184, 2007. [4] В. М. Бухштабер, Е. В. Корицкая, Функи. анализ и его прил., 41:3 (2007), 34-47. [5] V. M. Buchstaber, Combinatorics of simple polytopes and differential equations, http:/eprints.ma.man.ac.uk/1093/, 2008.

B. M. Бухштабер (V. M. Buchstaber)

Математический институт им. В. А. Стеклова РАН

E-mail: buchstab@mi.ras.ru
Представлено С. П. Новиковым Принято редколлегией 30.04 .2008 